\title{
Organic LEDs and solar cells united
}

\section{Organic donor-acceptor heterojunctions can show efficient electroluminescence and at the same time generate charges under photovoltaic operation.}

Wolfgang Brütting

Owing to fundamental principles of thermodynamics, in particular detailed balance between light absorption and emission, the performance of photovoltaic cells and electroluminescent diodes is linked by reciprocity relations. ${ }^{1}$ The more efficient a given semiconductor material is in lightemitting diodes (LED), the smaller is its bandgap-voltage offset in solar cells. ${ }^{2}$ However, this did not seem to be the case for organic semiconductors: in fact, research results in the last 25 years suggested that, in practice, photovoltaics (PV) and electroluminescence (EL) performance were mutually exclusive in organics-based devices. Writing in Nature Materials, Sascha Ullbrich and colleagues ${ }^{3}$ now show that this dichotomy can be reconciled with carefully designed organic donor-acceptor (D/A) heterojunctions.

Ever since the first appearance of efficient thin-film organic solar cells and LEDs in the late 1980s, heterojunctions between two unlike organic semiconductors have been key to device functioning. Different heterojunctions can be distinguished depending on the relative energy gaps of the two materials and their alignment. Of particular interest here is the alignment shown in Fig.1, known as type-II, where electrons on A and holes on D form Coulombically bound charge transfer (CT) states at the mutual interface. They can be considered as precursors for both free charges (Fig.1a), whose efficient generation determines the performance of a solar cell, and for photons emitted through radiative recombination (Fig.1b), the ultimate product of an electroluminescent device. Until recently, both fields of application seemed to be nonoverlapping in terms of materials used and even terminology.

Understanding of the mechanisms involving CT states at D/A interfaces has been recognized as key to the mastering of organic photovoltaics ${ }^{4-6}$. Interfacial CT states are formed on an ultra-fast timescale after photon absorption at one of the two materials, and need to dissociate to produce free carriers. Decay of this bound electron-hole pair, which can occur radiatively or nonradiatively, competes with the dissociation process, and is responsible for the offset between the energy of the CT state $\mathrm{E}_{\mathrm{CT}}$ and the energy corresponding to the open-circuit voltage $\mathrm{V}_{\mathrm{OC}}$ of the cell $e \Delta V_{t o t}:=E_{C T}-e V_{O C}$ (Fig.1c). ${ }^{7,8}$ Losses due to radiative decay are thermodynamically unavoidable, yet in most organic solar cells non-radiative decay processes dominate, causing large bandgap-voltage offsets in the order of $\Delta V_{\text {tot }} \sim 0.6 \mathrm{~V}$ or more.

Recently, such type-II interfaces attracted attention also in the field of organic LEDs. Because electron and hole do not sit on one and the same molecule, like in strongly bound excitons, but are spatially separated in a D/A pair, the exchange splitting between spin singlet and triplet states is much smaller and typically only of the order of the thermal energy. Thus, exploiting the principle of thermally activated delayed fluorescence to overcome spin-statistical limitations, 
such intermolecular CT states - commonly designated as exciplexes - can lead to efficient light emission across the visible spectrum. ${ }^{9,10}$ Due to their typically high non-radiative losses, however, one could hardly think that D/A pairs used in OPV may perform well as light emitters.

In their work, Ullbrich and colleagues show that light emission and charge generation are not mutually exclusive but can both be efficient processes, if the D/A pair fulfills certain conditions. The chosen combination of molecules (BF-DPB as donor with B4PYMPM as acceptor, see Fig. $1 \mathrm{~d}, \mathrm{e})$ shows yellow electroluminescence with an external EL quantum efficiency of $1.6 \%$, a factor of 10 less than the best known exciplex OLEDs but a $10^{4}$ factor higher than typical OPV systems ${ }^{11}$. The remarkable point, however, is that this device also has quite good photovoltaic performance parameters. In fact, when operated as a solar cell it delivers a $\mathrm{V}_{\mathrm{OC}}$ in excess of $2 \mathrm{~V}$, a high fill factor of $70 \%$ and a peak internal photon-to-charge carrier conversion efficiency over $80 \%$, which is comparable with other organic solar cells that absorb further in the near-infrared spectral region. Most importantly, the high $\mathrm{V}_{\mathrm{OC}}$ is due to very low non-radiative voltage losses $\Delta \mathrm{V}_{\mathrm{nr}}=0.1 \mathrm{~V}$, so that the overall $\Delta \mathrm{V}_{\text {tot }}$ amounts to $0.44 \mathrm{~V}$ only (Fig.1c) and comes close to inorganic PV materials, like Si or GaAs. This has also advantages in lighting applications, as the EL turn-on voltage relative to the CT energy is significantly lower than that observed in OLEDs that do not simultaneously function as efficient charge generating devices.

The secret behind this remarkable behavior is that in this particular system CT states at the D/A interface and free carriers in the bulk are in equilibrium ${ }^{12}$, meaning that the CT state binding energy $\left(E_{F C}-E_{C T}\right.$ in Fig. 1c) is so small that CT state dissociation occurs much faster than their decay. Then, contrary to common OLED knowledge, free electrons and holes after forming a CT state will not immediately undergo a transition to the ground state but have a high probability to re-dissociate again so that the whole process can start from the beginning. Only after several recombination-dissociation cycles will the CT state finally decay through competing radiative or non-radiative processes. By contrast, for strongly bound CT states every electron-hole encounter will directly lead to CT state decay. Such a device can still have high EL efficiency and, thus, high $\mathrm{V}_{\mathrm{OC}}$, but it will lose in fill factor and overall PV efficiency.

Another outcome of this work is that the related non-radiative voltage loss $\Delta \mathrm{V}_{\mathrm{nr}}$ follows the energy-gap law. This means that the radiative processes, and consequently the EL quantum efficiency, increase with $E_{C T}$, and this trend is followed not only by organic D/A systems used in photovoltaics (with CT energies in the near infrared) - as previously shown by this research team $^{13}$ — but for exciplex electroluminescence in the visible spectral range as well.

The importance of the work not only lies in a better understanding of loss processes in organic photovoltaics, which is mandatory to catch up with conventional technologies based on inorganic semiconductors, like Si or GaAs and the emerging lead halide perovskites. It might also change the design and selection criteria for materials for organic solar cells. For example, the viewpoint that photoluminescence quenching is a prerequisite for charge generation in organic PV may have obscured certain classes of materials not being investigated so far. This view has already been changing recently, when a significant boost of power conversion efficiency (reaching values over $15 \%$ now) has been achieved through the use of non-fullerene acceptors. ${ }^{14,15}$

The question is, of course, how generic this behavior is and what the main design rules for lowloss D/A heterojunctions are. Obviously structural order at the interface could help, as Ullbrich and coworkers suggest in their paper. One may further speculate that the reorganization energy 
between the neutral and charged state of a molecule also plays a role in deciding whether CT states and free carriers are in equilibrium. Clearly, more work is needed for predictive a-priori design of such low-loss D/A pairs. And, even though visible LEDs will never be great solar cells - simply because their energy gap is far off the optimum according to the Shockley-Queisser limit - there are potential applications for indoor light harvesting, UV-absorbing smart windows or multi-junction solar cells.

\section{Wolfgang Brütting,}

Institute of Physics, University of Augsburg, 86159 Augsburg, Germany

e-mail: bruetting@physik.uni-augsburg.de

\section{REFERENCES}

1. Rau, U., Phys. Rev. B 76, 085303 (2007)

2. Green, M.A., Prog. Photovolt: Res. Appl. 20, 472-476 (2012)

3. Ullbrich, S. et al., Nature Materials (this issue!)

4. Brédas, J. L., Norton, J. E., Cornil, J., \& Coropceanu, V. Accounts of Chemical Research, 42(11), 1691-1699 (2009).

5. Vandewal, K., Tvingstedt, K., Gadisa, A., Inganäs, O., \& Manca, J. V. Nature Materials, 8(11), 904 (2009).

6. Deibel, C., Strobel, T., \& Dyakonov, V. Advanced Materials, 22(37), 4097-4111 (2010).

7. Vandewal, K., Tvingstedt, K., Gadisa, A., Inganäs, O., \& Manca, J. V. Physical Review B, 81(12), 125204 (2010).

8. Gruber, M., Wagner, J., Klein, K., Hörmann, U., Opitz, A., Stutzmann, M., \& Brütting, W. Advanced Energy Materials, 2(9), 1100-1108 (2012).

9. Goushi, K., Yoshida, K., Sato, K., \& Adachi, C. Nature Photonics, 6(4), 253 (2012).

10. dos Santos, P. L., Dias, F. B., \& Monkman, A. P. The Journal of Physical Chemistry C, 120(32), 18259-18267 (2016).

11. Tvingstedt, K., Malinkiewicz, O., Baumann, A., Deibel, C., Snaith, H. J., Dyakonov, V., \& Bolink, H. J. Scientific Reports, 4, 6071 (2014).

12. Vandewal, K. Annual Review of Physical Chemistry, 67, 113-133 (2016).

13. Benduhn, J., Tvingstedt, K., Piersimoni, F., Ullbrich, S., Fan, Y., Tropiano, M., ... \& Barlow, S. Nature Energy, 2(6), 17053 (2017).

14. Qian, D., Zheng, Z., Yao, H., Tress, W., Hopper, T. R., Chen, S., ... \& Liu, X. K. Nature Materials, 17(8), 703 (2018).

15. Menke, S. M., Ran, N. A., Bazan, G. C., \& Friend, R. H. Joule, 2(1), 25-35 (2018). 


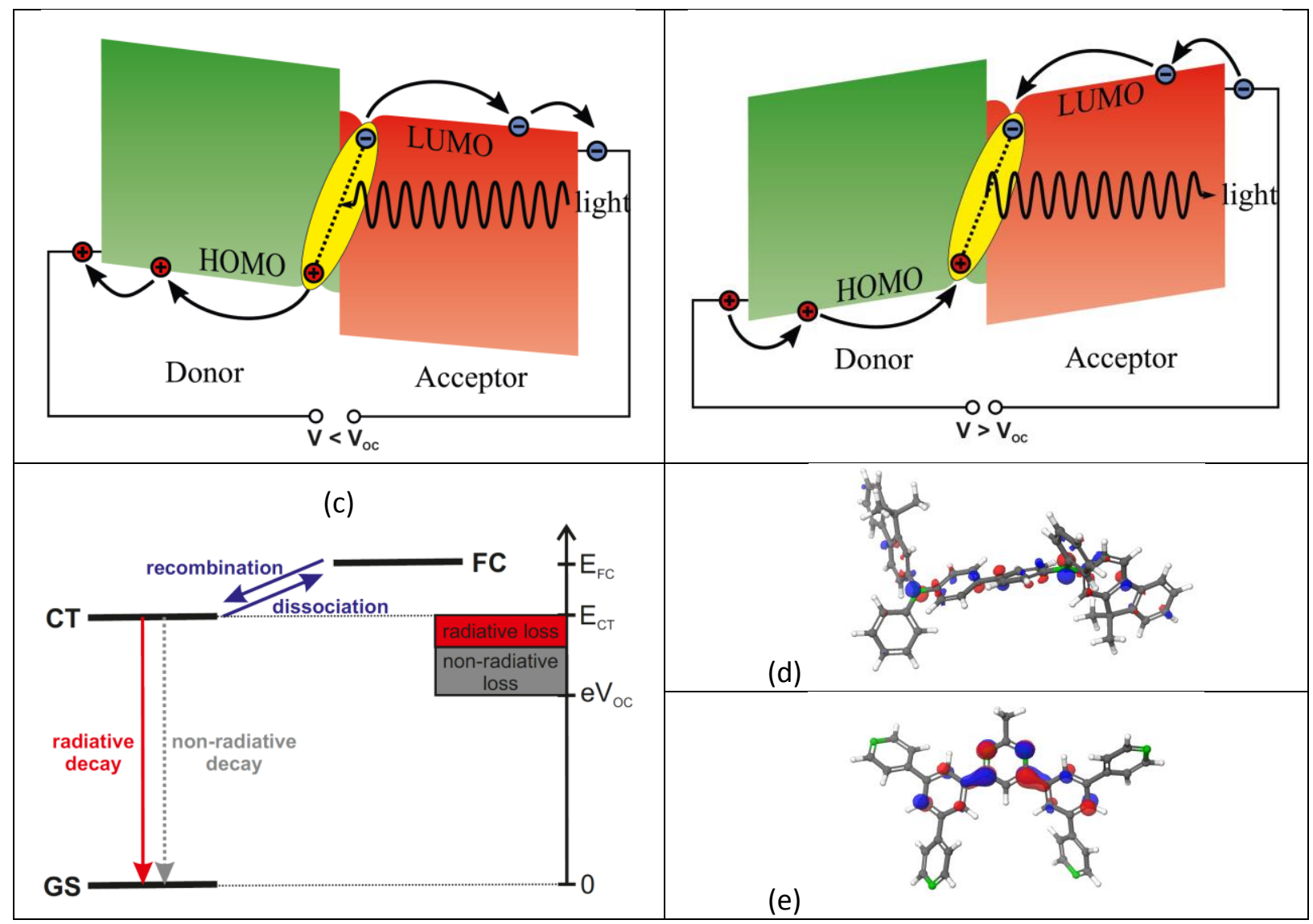

FIG. 1: Type-Il organic heterojunctions used as solar cells and LEDs, a-b, between an electron donor (D) and acceptor (A). An electron (blue sphere) sitting in the lowest unoccupied molecular orbital (LUMO) on A and a hole (red sphere) located in the highest occupied molecular orbital (HOMO) on D are Coulombically bound (yellow ellipse) at the interface, forming a charge transfer (CT) state. In a photovoltaic cell (a), free charges are generated by dissociation of CT states that are formed after light absorption (either directly or after charge transfer between D and A). In an LED (b) charge carriers are injected and form CT states upon recombination at the D/A interface, from where they can decay radiatively by emitting light or non-radiatively by producing heat. c, State diagram and relevant processes: GS - ground state, $C T$ - charge transfer state, $F C$ - free carriers. The difference between $E_{F C}$ and $E_{C T}$ is the $C T$ state binding energy, and between $\mathrm{E}_{\mathrm{CT}}$ and $\mathrm{eV}_{\mathrm{OC}}$ the energy (or voltage) loss in a solar cell, which is composed of a radiative and a non-radiative contribution, according to their relative strengths. If the CT state binding energy is small, free charges and CT states are in equilibrium and will undergo many recombination-dissociation cycles (blue arrows) before they finally decay to the ground state. d-e, Chemical structures of exemplary donor (BF-DPB, d) and acceptor (B4PYMPM, e) molecules. For BF-DPB the HOMO and for B4PYMPM the LUMO orbitals are shown, respectively. Carbon atoms are shown in grey, hydrogen in white and nitrogen in green. Blue and red color indicates positive and negative sign of the respective wavefunction. 